\title{
Fishery externalities and biodiversity: Trade-offs between the viability of shrimp trawling and the conservation of Frigatebirds in French Guiana
}

\author{
Vincent Martinet ${ }^{\mathrm{a},{ }^{*}}$ and Fabian Blanchard ${ }^{\mathrm{b}}$ \\ a Economie Publique, UMR INRA-AgroParisTech, 78850 Thiverval-Grignon, France \\ ${ }^{\mathrm{b}}$ Ifremer, France \\ *: Corresponding author : Vincent Martinet, email address : vincent.martinet@grignon.inra.fr
}

\begin{abstract}
:
Sustainable management of natural resources, and in particular fisheries, must take into account several conflicting objectives. This is the case in the French Guiana shrimp fishery for which profitability objectives imply a reduction in the fishing activity. On the one hand, this fishery has negative externalities on marine biodiversity due to discards. On the other hand, this fishery has positive externalities on the economy of the local community and interestingly enough on a protected seabird species in the area (the Frigatebird that feeds on discards). In this paper, we examine the viability of that system considering two sustainability objectives: an economic objective in terms of the profitability of the fishing activity, and a conservation objective in terms of the Frigatebird population. For that purpose, we have developed a dynamic model of that bioeconomic system and study here the trade-offs between the two conflicting objectives. It provides a means to quantify the necessary give and takes involving the economic and ecological objectives that would ensure a viable management solution. Our study confirms the relevance of the viability approach to address natural resource management issues, which should lead to the development of new tools for the arbitration of conflicting sustainability objectives. In particular, such tools could be used as a quantitative basis for cost-benefit analysis taking into account environmental externalities.
\end{abstract}

Keywords: Bio-economic modeling; Viability; Sustainability; Fishery externalities; Species conservation

JEL classification codes: Q22; C61 
2 Fishery activities generate externalities on biodiversity. On the one hand, there 3 are numerous negative externalities linked to fishery discards in terms of bycatch species and loss of marine biodiversity. Indeed, eliminating discards is currently a major political objective (CEC, 2007). On the other hand, one potential positive externality of these discards is that they may play a fundamental role in marine bird feeding (Furness, 2003). According to Furness (1999), reducing fishery discards may dramatically reduce some seabird populations. This is also the case when discards are reduced due to an adjustment of fishing activities related to the economic context. For example, the prior level of fishing activity of the French Guiana shrimp fishery is no longer economically viable given the present prices, costs, and amount of subsidies. The recent reduction in that fishing activity has resulted in a high rate of Frigatebird chick mortality and has triggered a conflict between the ecological objective of the Frigatebird conservation program off the caribbean coast of French Guiana and the economic objective of the fishery. Managing fishery activities in a sustainable way must thus take into account conflicting objectives of ensuring economic viability while preserving marine and bird biodiversity.

In ecological economics, it is now recognized that multicriteria modeling, and especially the viability approach (Aubin, 1991), are well-suited to address sustainability issues (De Lara and Doyen, 2008). The aim of viability approach is to study the consistency between a dynamic model and a set of constraints. It consists in defining the conditions for the constraints to be satisfied at all times. In particular, thanks to the viability approach, it is possible to characterize the dynamics of a bio-economic system in terms of its capacity to achieve, in the long-run, sustainability objectives represented by ecological and economic constraints. Béné et al. (2001), Doyen and Béné (2003) and Eisenack et al. (2006) have used the viability approach to investigate natural resource management issues. Cury et al. (2005) have argued that the application of the viability approach is relevant for an ecosystem management of fisheries. Indeed, the viability of fisheries has recently been studied by Doyen et al. (2007), Martinet et al. (2007) and Chapel et al. (2008), among others.

Viability studies usually account for constraints with given levels. In this study, we set out to investigate a way to account for potential interactions between constraint levels. That would provide much needed information about tradeoffs between sustainability objectives. In the example of the French Guiana shrimp fishery and Frigatebirds, it would allow us to describe the trade-offs 
between ensuring the viability of the shrimp trawling and maintaining the bird population which feeds on fishery discards.

To this end, we have developed a dynamic bioeconomic model of a fishery that generates discards which are a source of food for a bird population. We account for two sustainability objectives (represented by constraints): an economic constraint on the profitability of the fishing activity, and a conservation constraint of the bird population. By extending the viability approach, we examine how these sustainability objectives are compatible one with respect to the other, and if there are trade-offs between both viability constraint levels. In other words, we are dealing with how to cope with two seemingly different objectives at the same time, and more specifically with the give and take in the level of constraints that must be worked out to be able to reach these objectives.

The paper is organized as follows. In Section 2, we present a model based on the Guianese shrimp fishery. In Section 3, we address the co-viability issue of achieving at the same time economic and ecological objectives in a dynamic way. In Section 4, we extend the viability approach by describing the tradeoffs between economic and biological objectives. We also define the economic conditions that are necessary (including the minimum amount of subsidies) if the Guianese fishing activity is to be viable while maintaining a targeted Frigatebird population level. In Section 5, we discuss the pertinence of the extended viability approach as well as its usefulness as a tool that provides a well-grounded basis for arbitration between conflicting sustainability objectives. Parameter values and mathematical proof are provided in the appendix.

\section{A model of fishery interacting with a seabird population}

\subsection{The French Guiana case study}

The shrimp fishery in French Guiana is composed of trawlers fishing for shrimp on the continental shelf. Two main species are involved: Farfantepeneus subtilis and F. brasiliensis. Only F. subtilis was accounted for. It is the species caught the most often and, since the eighties, it has been thoroughly investigated by Ifremer (the French institute of research for the exploitation of the sea) providing a solid knowledge of the population and exploitation dynamics. From a historical point of view, the economic dynamics of the fishery have been characterized by a decrease in the fishing activity for profitability purpose. In 
turn, the amount of catch has dramatically decreased, and actually it is about half the Maximum Sustainable Yield (MSY). This decrease in fishing activity implied a decrease in discard.

The Frigatebird Fregata magnificens population in French Guiana is the most important colony of this seabird species from North Brazil to Venezuela. The colony is located in a natural reserve on "Le Grand Connetable", a small island which makes survey easy. Their are exceptional birds, because of their low reproduction rate, their long period of parental care (the longest of any bird), and their long life spend (more than 30 years.) (Weimerskirch et al., 2003). Before the development of the shrimp fishery (and associated discards), the Frigatebird population was stable, with about 180 nesting couples succeeding in raising their chick. Since it is not possible here to represent the Frigatebird population in a dynamic way in our case (sufficient long-run data is not yet available to assess the dynamics), the number of breeding bird couples serves as a proxy for the Frigatebird population.

Calixto-Albarran and Osorno (2000) have found a correlation between the variety of fish in the diet of Frigatebird population on Isla Isabel (off the Pacific coast of Mexico) and species discarded by prawn-fishing trawlers in the area, thereby assuming an opportunistic feeding during nesting period. Based on personal field observation that found 120 Frigatebirds feeding on the discard of a sole shrimp trawler, the same correlation is assumed to hold for the Guianese population. A strong correlation has been also observed between chick mortality during breeding and periods of declining fishing effort (and associated decreasing discards) within the area of bird foraging (unpublished data). Until recently, the decrease in discard had no impact on the Frigatebird population, but the ongoing decline of the fishery and the associated observed mortality of chicks now jeopardize the conservation program. In the 2007 economic context, some of the 639 surveyed couples were not able to feed their chick. Understanding the interactions between economic dynamics and the conservation objectives is therefore necessary. For that purpose, we develop a bioeconomic model of the fishery.

\subsection{The bioeconomic model}

We consider a single stock fishery, characterized every year $t$ by the biomass $B_{t}$ of the resource stock (shrimp in our case study). The dynamics of the bioeconomic system is controlled by the fishing effort $E_{t}$, following Clark (1985). The global harvest is defined by $H_{t}=q B_{t} E_{t}$, where the constant parameter 
$q$ represents the catchability of the resource. Using a discrete time version of the "logistic model" to represent the growth function of the shrimp stock, the dynamics of the resource stock is given by

$$
B_{t+1}=B_{t}+R\left(B_{t}\right)-H_{t}=B_{t}+r B_{t}\left(1-\frac{B_{t}}{B_{\text {sup }}}\right)-q B_{t} E_{t}
$$

where $B_{\text {sup }}$ is the carrying capacity of the ecosystem, and $r$ the natural growth rate of the resource stock $(r<1)$.

The fishery is characterized by profit given as

$$
\pi_{t}=(p+\tau) H_{t}-c E_{t}=(p+\tau) q B_{t} E_{t}-c E_{t}
$$

where $p$ is an exogenous resource price, $\tau$ is a production subsidy and $c$ is the per effort unit cost.

This fishery generates discards of bycatch species. These discards depend on the fishing effort $E_{t}$. A part of these discards is used by seabirds to feed themselves and to feed newborns during the breeding season (Frigatebirds in our case study). We define the quantity of discards available for birds as $D_{t}=d E_{t}$, where $d$ is a discard constant, i.e., the quantity of discarded biomass that birds can eat per unit of fishing effort. An important point is that the discards are made up of bycatch species (fish, squid, starfish, crabs, jellyfish), hence not proportional to the catches of the targeted species (to the shrimp biomass) but to the fishing effort (the overall number of trawler's haul).

We are interested in the number of Frigatebird couples that make a nest and find enough food to raise the chick until it can leave the nest. We assume the following relationship between discards and Frigatebird nests

$$
F_{t}=s D_{t}+F_{0}
$$

where $F_{0}$ is the number of Frigatebird couples that raised a chick successfully before fishing began in the area and there was no discard. $s$ is a constant parameter describing the effect of the new food source provided by discards. 


\subsection{The viability constraints}

In the present analysis, we will focus on two viability constraints.

On the one hand, the economic viability of the shrimp fishery depends on its profit that has to be positive, i.e., $\pi_{t} \geq 0$.

Defining the catch per unit of effort $h_{t}=H_{t} / E_{t}$ (for $E_{t}>0$ ), and using the profit definition (eq. 2), leads to the following

$$
\pi_{t} \geq 0 \quad \Rightarrow \quad h_{t} \geq \frac{c}{p+\tau}
$$

The catch per unit of effort $h_{t}=q B_{t}$ must therefore be greater than a threshold $h_{\min }=\frac{c}{p+\tau}$ for the fishing activity to be profitable. This threshold depends on the economic context (resource price, subsidies level, and cost structure). The viability constraint representing that economic objective is thus defined as

$$
h_{t} \geq h_{\min } .
$$

On the other hand, an ecological objective is to protect the Frigatebird population. For that purpose, a minimum number of couples able to feed chicks is targeted. The viability constraint representing this ecological objective is thus defined as

$$
F_{t} \geq F_{\min }
$$

We aim at defining bioeconomic configurations that make it possible to satisfy both the constraints in a dynamic way.

\section{Co-viability analysis}

\subsection{The viability framework of analysis}

To develop our analysis, we have adopted the viability approach. The purpose of our analysis is to determine if there are inter-temporal viable exploitation decisions $E($.$) that make it possible to satisfy both the economic objective$ 
(eq. 4) and the conservation objective (eq. 5), at all times $t \geq t_{0}$, given the dynamics of the fishery (eq.1).

The approach is based on the definition of states $B$ and controls $E$, satisfying dynamics (1) resulting in trajectories that respect the constraints (4) and (5). We define the set of states $B$ from which there exist inter-temporal decisions resulting in viable trajectories. Formally, this set, called the viability kernel of the problem, is defined by

$$
\operatorname{Viab}\left(h_{\text {min }}, F_{\text {min }}\right)=\left\{\begin{array}{l|l}
B_{0} & \begin{array}{l}
\exists E(.) \text { and } B(.) \text { starting from } B_{0} \\
\text { satisfying dynamics (1) } \\
\text { and constraints (4) and (5) } \forall t \geq t_{0} .
\end{array}
\end{array}\right\}
$$

The viability kernel of our problem is determined in subsection 3.2. From any state inside the viability kernel, there exists at least one viable decision driving the dynamic system on a viable trajectory, i.e., a trajectory that respects the constraints at all times. On the contrary, if the state is outside the viability kernel, or if the trajectory leaves it, there are no decisions making it possible to respect the constraints forever, and at least one of the constraints will be violated within a finite time. It means that if state $B$ is not in the viability kernel, the viability objectives can not be achieved intertemporally. As a consequence, if the viability kernel is empty, there are no bioeconomic states of the fishery that allows the satisfaction of both economic and ecological constraints in the long run. It means that the sustainability objectives are too ambitious and could never be achieved in the long run, whatever the initial condition of the system. To avoid such an unviable situation, one can relax one of the constraints. We will use that approach in Section 4.

From a mathematical point of view, the viability kernel is an invariant set. It is the biggest set of states such that from any of those states there are admissible decisions resulting in dynamics that both satisfy the viability constraints and remain within the set. This means that from any viable state, at least one dynamic path remains within the viability kernel. Viable decisions are thus defined such that the viability constraints are satisfied and the state of the system stays within the viability kernel. 
where

$$
\underline{B}\left(h_{m i n}\right)=\frac{h_{\min }}{q} .
$$

${ }_{188}$ The associated viable decisions $E^{v i a b}$ must satisfy conditions

$189 \quad \underline{E}\left(F_{\text {min }}\right) \leq E^{\text {viab }} \leq \bar{E}\left(B, h_{\text {min }}\right)$, where

$$
\underline{E}\left(F_{\text {min }}\right)=\frac{F_{\text {min }}-F_{0}}{s d}
$$

190

and

$$
\bar{E}\left(B, h_{\min }\right)=\frac{1}{q}\left(1+r\left(1-\frac{B}{B_{\text {sup }}}\right)-\frac{h_{\text {min }}}{q} \frac{1}{B}\right)
$$

191 If $\left(h_{\min }, F_{\min }\right)$ do not satisfy condition $(7)$, the viability kernel is empty $192\left(\operatorname{Viab}\left(h_{m i n}, F_{m i n}\right)=\emptyset\right)$.

193 When it is not the empty set, the viability kernel is as represented on Fig.1 


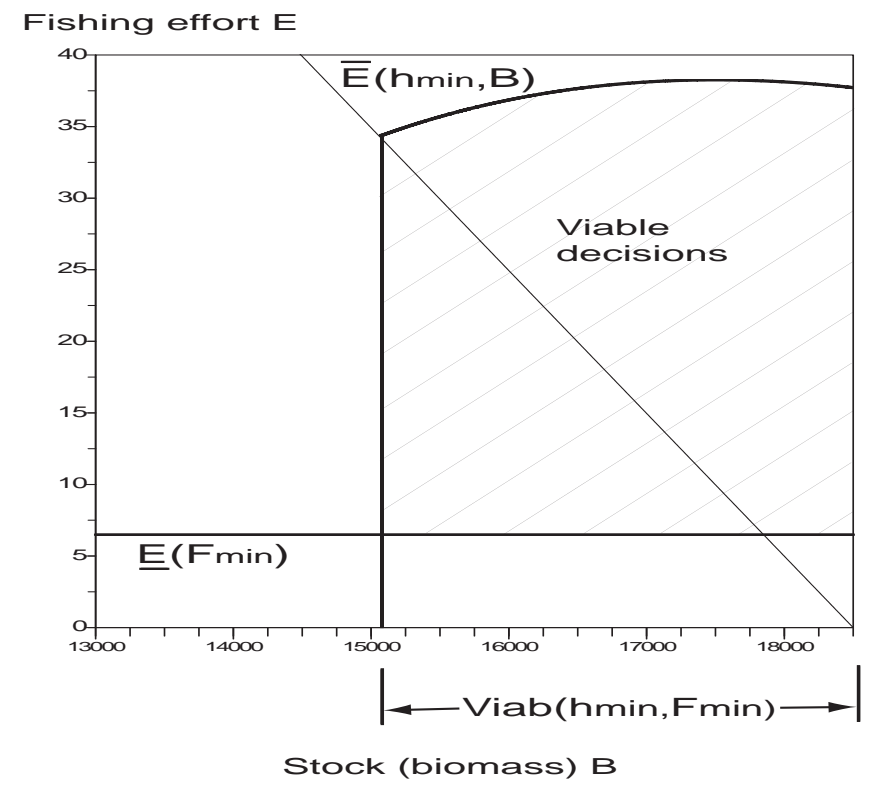

Fig. 1. Viability kernel (stock biomass) associated with economic constraint $h_{\min }$ and conservation objective $F_{m i n}$, and associated viable decisions (fishing effort).

\subsection{Sensitivity analysis}

When the viability kernel is not empty (i.e., if condition (7) holds), it expression depends on the constraint threshold $h_{\min }$ (see eq. 8). Moreover, viable decisions depend on both $h_{\min }$ and $F_{\min }$ (see eqs. 10 and 11).

In our viability problem, the economic constraint (4) corresponds to a viability condition depending on the economic context. This context may change (if prices, subsidies, or costs change), resulting in a change in the viability kernel. In a similar way, the ecological constraint (5) is an ecological objective that may be adjusted. We provide here a sensitivity analysis of the results with respect to the levels of the constraints.

From eq. (9), one can see that $\underline{B}\left(h_{\min }\right)$ increases with $h_{\min }$, which means that the higher the economic constraint (the worse the economic context), the higher the induced stock constraint, and then the smaller the viability kernel.

Fig. 2 represents this result. We consider two economic contexts, $h_{\min } 1$ and $h_{\min } 2$, with $h_{\min } 2>h_{\min } 1$ meaning that the economic context is more favorable to the fishery in case 1 (higher price and/or subsidy, and/or lower costs); and two ecological objectives $F_{\min } 1$ and $F_{\min } 2$, with $F_{\min } 2>F_{\min } 1$, meaning 


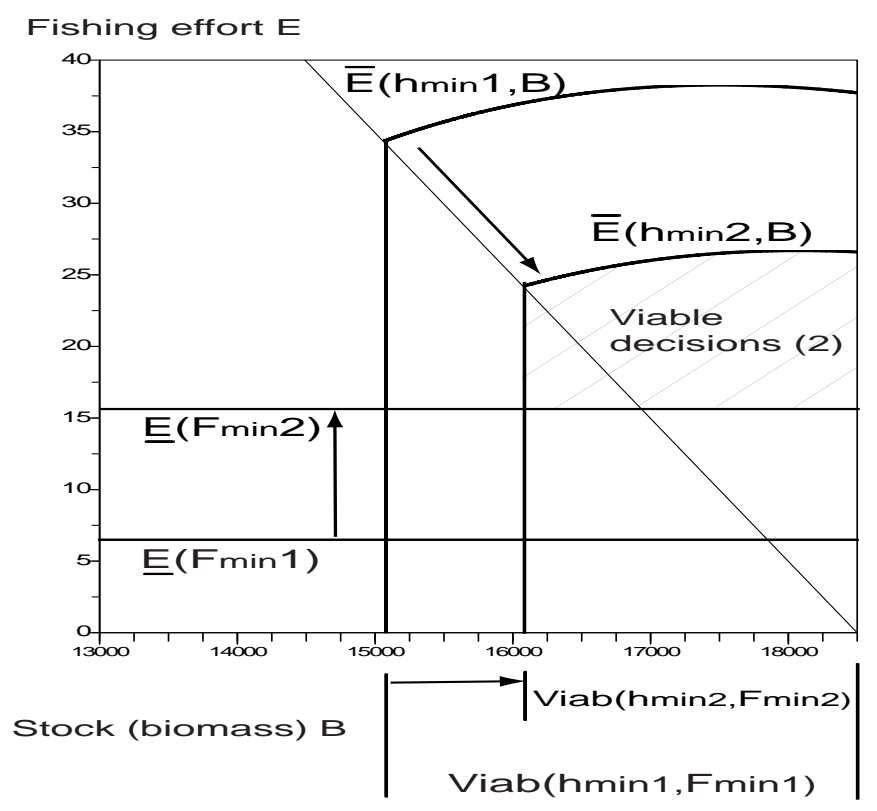

Fig. 2. Sensitivity of the Viability kernel $\operatorname{Viab}\left(h_{\min }, F_{\min }\right)$ to the constraints levels. $h_{\min } 2>h_{\min } 1$ and $F_{\min } 2>F_{\min } 1$. The higher the constraints, the smaller the kernel and the more reduced the associated viable decisions.

In Fig.2, the higher the economic constraint level, the smaller the viability kernel. Moreover, as the threshold $\underline{E}\left(F_{\min }\right)$ linearly increases with respect to the constraint level, the viable decisions are reduced when the ecological objective increases.

If the conservation objective is too high, it is not possible to reach the ecological objective for the given economic constraint; the viability kernel is empty (no state makes it possible to satisfy both the constraints over time). From dynamical perspective, if the economic context is degraded (i.e., if $h_{\min }$ increases) the maximum viable effort $\bar{E}(\underline{B})$ decreases, inducing a lower potential conservation of the bird population. There is thus a trade-off between economic viability and ecological conservation. In the next section, we study that point in more detail.

As regards condition (7), we can say that the conservation objective must be lower than a threshold depending on the economic context. The higher $h_{\min }$ is, the lower $F_{\min }$ must be for the viability kernel not to be empty. As we should see, this condition will play a crucial role in the trade-offs between sustainability objectives. 


\section{Trade-off between sustainability objectives}

In this section, we discuss the consequences of the economic viability of the fishery on the conservation objective of the Frigatebird population. We first provide an analysis describing trade-offs between the economic objective and the biodiversity conservation objective. We then define the economic incentive that would make it possible to reach a given conservation objective, when it is not possible in the initial economic context, and the associated cost.

\subsection{Set of reachable objectives}

In a given economic context (i.e., for $h_{\min }$ corresponding to given prices, costs and subsidy levels), it would be interesting to know how large a Frigatebird population can be in the long run. To obtain this information, we compute the maximum conservation objective for which the viability kernel is not empty. We define the maximum reachable conservation objective with respect to $h_{\min }$ as follows.

$$
\mathcal{F}\left(h_{\text {min }}\right)=\max \left\{F_{\text {min }} \mid \operatorname{Viab}\left(h_{\text {min }}, F_{\text {min }}\right) \neq \emptyset\right\}
$$

The non-emptiness of the viability kernel depends on relationship (7). The maximum level $F_{\min }$ that satisfies this condition is

$$
\mathcal{F}\left(h_{\text {min }}\right)=\frac{r s d}{q}\left(1-\frac{h_{\text {min }}}{q B_{\text {sup }}}\right)+F_{0}
$$

According to our calculation, given the 2007 economic context, the maximum number of Frigatebird couples expected to successfully breed is (around) $\mathcal{F}\left(h_{\text {min }}\right)=478$ couples, meaning that some of the 639 Frigatebird couples surveyed in 2007 would lose their chick during the nesting period.

We have exhibited a trade-off between the economic constraint $h_{\min }$ and the ecological constraint $F_{\min }$. Achievable conservation objectives must satisfy $F_{\text {min }} \leq \mathcal{F}\left(h_{\text {min }}\right)$. To increase the level of one of the constraints above the threshold given by compatibility relationship (13), it is necessary to reduce the level of the other.

To provide more information about trade-offs between sustainability objec- 
tives, in the following section we examine the give and takes between the conservation objective $F_{\min }$ and the economic objective $h_{\min }$.

\subsection{Equivalent economic incentives: One of the costs of biodiversity conser- vation}

In our case study, the economic constraint is defined by the economic context. This constraint can be modified by changing the subsidy level (increasing it or decreasing it). The ecological objective is more flexible as it is a chosen target. It can be adapted in order to have a non-empty viability kernel.

It is possible to define the necessary economic conditions to be able to reach a given conservation objective $F_{\text {min }}$, that is to say to look for the economic conditions resulting in a $h_{\min }$ such that the viability kernel is not empty. For this purpose, we define the reciprocity of relationship (13), i.e., the maximum level of $h_{\min }$ that is compatible with an ecological constraint $F_{\min }$ :

$$
\mathcal{H}\left(F_{\text {min }}\right)=\max \left\{h_{\text {min }} \mid \operatorname{Viab}\left(h_{\text {min }}, F_{\text {min }}\right) \neq \emptyset\right\}
$$

We have

$$
\mathcal{H}\left(F_{\text {min }}\right)=q B_{\text {sup }}\left(1-\frac{q\left(F_{\text {min }}-F_{0}\right)}{r s d}\right)
$$

This level corresponds to the worst economic context compatible with the Frigatebird population objective $F_{\min }$. If the economic situation is worse, i.e., if the economic proxy $h_{\text {min }}=\frac{c_{t}}{p_{t}+\tau_{t}}$ is higher than the threshold $\mathcal{H}\left(F_{\text {min }}\right)$, the viability constraints cannot be satisfied. It means that to be able to reach a conservation objective $F_{m i n}$, it is necessary to modify the viability constraint $h_{\text {min }}$ (by changing the economic context, adjusting the subsidy level) so that the viability kernel is not empty.

As the level of the economic proxy depends on the economic context, one can compute the equivalent shrimp price (including subsidies) at which a given ecological viability objective $F_{\min }$ would be reachable ${ }^{1}$

1 The same kind of analysis could have been done on cost structure $c_{t}$ with a discussion on the evolution of fishing costs, such as oil, and potential specific subsidies. 


$$
\begin{aligned}
h_{\text {min }} \leq \mathcal{H}\left(F_{\text {min }}\right) & \Leftrightarrow \frac{c}{(p+\tau)} \leq q B_{\text {sup }}\left(1-\frac{q\left(F_{\text {min }}-F_{0}\right)}{r s d}\right) \\
& \Leftrightarrow(p+\tau) \geq \frac{c}{q B_{\text {sup }}}\left(\frac{1}{1-\frac{q\left(F_{\text {min }}-F_{0}\right)}{r s d}}\right)
\end{aligned}
$$

To achieve the conservation objective $F_{\text {min }}$, a minimum fishing activity is needed. That level of fishing activity is profitable only if the selling price (price plus subsidy) is higher that the threshold defined in eq. (16). As this selling price includes the exogenous market price $p_{t}$ and the subsidy $\tau_{t}$, the minimum level of the subsidy that will result in a non-empty viability kernel is defined as

$$
\tau^{*}\left(F_{\text {min }}\right)=\frac{c}{q B_{\text {sup }}}\left(\frac{1}{1-\frac{q\left(F_{\min }-F_{0}\right)}{r s d}}\right)-p
$$

This level of subsidy ${ }^{2}$ can be interpreted as follows: If a conservation objective $F_{\text {min }}$ higher than the value $\mathcal{F}\left(h_{\text {min }}\right)$ (with $\left.h_{\text {min }}=\frac{c_{t}}{p_{t}+\tau_{t}}\right)$ is to be reached, it is necessary to provide an economic incentive at a level $\tau^{*}$, instead of the initial level $\tau_{t}$. In a symmetric way, note that when the viability kernel is not empty (when economic constraint $h_{\text {min }}$ is lower than $\mathcal{H}\left(F_{\text {min }}\right)$ ), it is possible to reduce subsidy level from $\tau_{t}$ to $\tau^{*}$ in order to reduce bycatch while still satisfying the conservation objective $F_{m i n}$.

By construction, if $\tau=\tau^{*}\left(F_{\text {min }}\right)$, then $h_{\text {min }}=\mathcal{H}\left(F_{\text {min }}\right)$ (which expression is given by eq. 15). It is possible to compute the minimum cost of such an incentive program by multiplying the subsidies level $\tau^{*}$ by the minimum quantity of shrimp $\underline{H}$ harvested in the viability kernel, i.e., at the equilibrium state $\underline{B}\left(h_{\text {min }}\right)$ which is associated to effort $\underline{E}\left(F_{\text {min }}\right)$. It reads $\underline{H}=q \underline{B}\left(h_{\text {min }}\right) \underline{E}\left(F_{\text {min }}\right)$. Moreover $h_{\min }=\underline{H} / \underline{E}$, which leads to $\underline{H}=\mathcal{H}\left(F_{\text {min }}\right) \underline{E}$. As from eq.(17) $\tau^{*}=\frac{c}{\mathcal{H}\left(F_{\text {min }}\right)}-p$, the expression of the minimum total subsidy cost $\mathcal{S}\left(F_{\text {min }}\right)$ can be written as

$\overline{2}$ From an economic point of view, a negative subsidy is a tax. In the following analysis, this case is not excluded. Our result can also be interpreted as follows: what could be the maximum tax level (in order to reduce fishery's activity and bycatch level) compatible with a given conservation objective of the Frigatebird population. 


$$
\begin{aligned}
\mathcal{S}\left(F_{\text {min }}\right) & =\left(\frac{c}{\mathcal{H}\left(F_{\text {min }}\right)}-p\right) \mathcal{H}\left(F_{\text {min }}\right) \underline{E}\left(F_{\text {min }}\right) \\
& =\left(c-p q B_{\text {sup }}\left(1-\frac{q\left(F_{\text {min }}-F_{0}\right)}{r s d}\right)\right) \frac{F_{\text {min }}-F_{0}}{s d}
\end{aligned}
$$

Eq. (19) only depends on exogenous parameters and on the viability target $F_{m i n}$. It is a parabola which is equal to zero when the target $F_{m i n}$ is the natural level $F_{0}$. Fig. 3 represents that cost with respect to the viability constraint $F_{\text {min }}$.

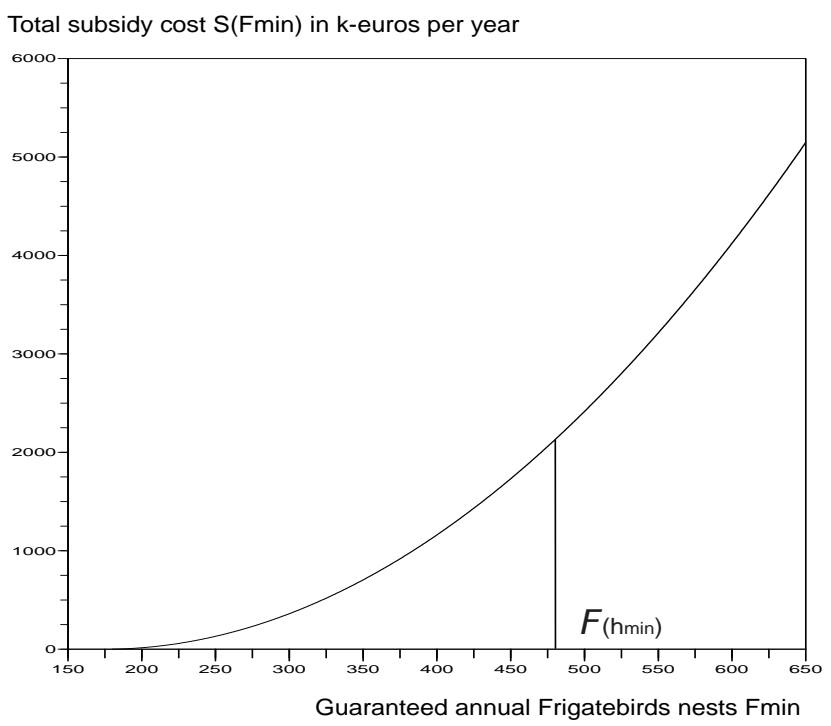

Fig. 3. Total annual cost of a subsidy program $\mathcal{S}\left(F_{\min }\right)$ with respect to the Frigatebirds conservation objective $F_{\text {min }}$. As a benchmark, $\mathcal{F}\left(h_{\text {min }}\right)$ is the maximum conservation objective that is reachable in the present economic context (without modifying the subsidy level).

\section{Conclusion}

Several seabird species feed on the discards of fisheries. If the fishery collapses for economic reasons, the bird population could decline. In this paper, we focus on the the particular and unusual relationship between the subsidized Guianese shrimp fishery and the protected Frigatebird population. The recent decline in the trawling activity has been correlated with an increased Frigatebird chicks mortality rate. Using a bioeconomic model describing the dynamics of the shrimp fishery and its interactions with the Frigatebird population, we 
have accounted for two apparently conflicting sustainability objectives represented by constraints: an economic constraint on the profitability of the fishing activity, and a conservation constraint of the bird population. By extending the viability approach, we have examined how these sustainability objectives are compatible one with respect to the other, and if there are trade-offs between both viability constraint levels. More specifically, we have been able to investigate the give and takes in the level of constraints that must be worked out to be able to reach these objectives.

In our case study, the maximum Frigatebird population that can be conserved depends on the fishing activity, that is limited by the economic context of the fishery. It would be possible to conserve a larger population if subsidies were granted to increase the shrimp trawling. Thanks to our approach, we are able to determine the lowest level of subsidies needed to ensure the economic viability of the fishery while maintaining a targeted Frigatebird population. Moreover, we came to understand that if that level is lower than the current amount of subsidies, it would be possible to further reduce the subsidy level without arming the bird population. On the contrary, if that level is higher than the current level, it would amount to what we call an "extra-cost". In this case, it appears that the managers of the conservation program should be aware of this when they define their ecological objective in terms of population number. To sum up, the objectives of a conservation program (within a protected area) cannot be defined without taking into account potential interactions with ecological and economic dynamics outside the protected area.

Our study confirms the relevance of the viability approach to account for ecological and economic objectives in the case of natural resources management issues. Applying this approach makes it possible to define the bioeconomic conditions for several objectives, represented by constraints and given target levels, to be achievable at all times. By extending the viability approach to account for the give and takes of the constraint levels, it is possible to determine the set of achievable objectives and pinpoint trade-offs between sustainability targets. This extended approach would provide policymakers with thorough knowledge of all the possible achievable objectives, including trade-offs between conflicting ones, and therefore provide a well-grounded basis for arbitration. In our study, the quantitative description of the trade-offs between sustainability objectives could be a starting point for a broader environmental economic analysis aiming to define the socially optimal level of an incentive program. The cost of fishery subsidies has to be compared to i) its benefits in terms of social externalities (communities support and employment) and ecological positive externalities (Frigatebird conservation), and to ii) its costs 


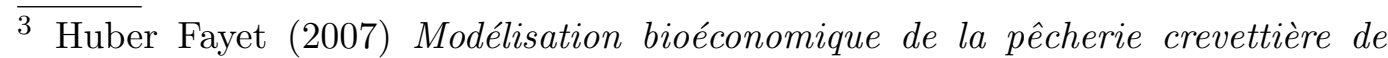
Guyane Française, Master thesis, under the supervising of Christian Chaboud (IRD).

Acknowledgments This paper was prepared as part of the "CHALOUPE" research project, funded by the French National Research Agency under its "Biodiversity" program 2005. We thank Philippe Vendeville (Ifremer) for advice on shrimp population dynamics, Julien Semelin (manager of the natural reserve "Le Grand Connetable") for access to data on the bird population, and Christian Chaboud (IRD) who led the economic surveys. We also thank the directors of the fishing companies for their cooperation. We are most grateful to Olivier Thbaud (Ifremer) for his stimulating discussion, the two anonymous referees for all their insightful comments, and Suzette Tanis-Plant for fruitful discussions and editorial advice in English. All remaining errors are own.

\section{A Appendix}

\section{A.1 Parameter values}

We present here the way parameters were obtain, and the hypothesis underlying our model.

Economic and biological parameters for the shrimp fishery Biological parameters of the shrimp fishery were estimated using LPUE series (landings per unit of effort) as an index of abundance. These LPUE were computed using information from fishing companies log books on fishing time and landings. We used non linear parameter estimation techniques to find the best fit of the predicted LPUE, given the observed LPUE. The fitting criterion is the minimization of the square deviation, using the methods provided by Hilborn and Walters (1992).

Economic parameters (costs, prices, and subsidies) in the 2007 economic context were computed by Huber Fayet. ${ }^{3}$ In 2007, the economic context was $p_{2007}=7$ k-euros per ton ; $\tau_{2007}=1.1 \mathrm{k}$-euros per ton $; c_{2007}=641.893 \mathrm{k}$-euros. These values were defined from economic surveys carried out in May 2007 within the Chaloupe project, in the three major shrimp fishing companies. In the 2007 economic context, the minimum catches per unit of effort ensuring profitability was 79.246 tons of shrimps per effort unit (it corresponds to a resource stock $B=16,074$ tons). The RD). 
4 Blanchard F. and Semelin J. (2008) Impact of fishery discards on the population dynamics of a tropical marine bird species (Fregate Magnificiens) in French Guiana: when fishermen strike affects the bird reproduction success. Unpublished work. Chaloupe working paper.

Frigatebird and fishery interaction parameters The parameters of the interaction between the Frigatebird population and fishery discards were obtained by Fabian Blanchard and Julien Semelin. ${ }^{4}$ We explain here the basic idea underlying relationship (3).

In our model, the fishing effort unit has been defined as the total effort developed by a vessel during one year. Taking into account the maximum individual size of a fish a Frigatebird is capable of swallowing, a trawler haul generates $11.2 \mathrm{~kg}$ of discarded biomass the birds can feed on. Computing the mean number of days at sea per vessel and per year (which is quite constant around 258 days at sea per vessel per year in our case study, as climatic conditions in this area are quite similar from one year to another), and accounting for two trawling haul per day, each fishing effort unit $E$ generates $d=5.78$ tons of discards available as a source of food for birds per year. Given the facts that the bird species has a long life time and a late maturity age, and that data on the population size are available only for recent years, it is not possible to model, according to current knowledge, the population dynamics and the influence of discard on it. We thus have to make some strong assumption on the relationship between discard and reproduction success. The number of nests in natural conditions (the one observed before the development of the fishery in the 60 's) is about 180 couples succeeding in reproduction each year. This leads to our reference population $F_{0}$. Given recent data on discard and Frigatebird population survey, chick mortality occurs every time the quantity of discards decreases below $336 \mathrm{~kg}$ per couple. In particular, 215 tons of discards made it possible to ensure the viability of the 639 nests surveyed in January 2007. We assume that discards have a linear effect on reproduction success, and consider two reference points: 180 couples are viable with no discard, 639 couples are viable with 215 tons of discards. It gives us a slop of 2.135 for the linear relationship linking bird couples to discard. Hence the parameters of eq.(3).

The 2007 survey of Frigatebird population leads to an evaluation of the population size of 639 couples (two adults and a nest). A total conservation objective would results in a threshold $F_{\min }=639$ (this objective would require to maintain a quantity of discards available for feeding of 215 tons each year). 
The following table gives the parameters value for our case study.

\begin{tabular}{|c|c|c|c|}
\hline Parameter & name & value & (units) \\
\hline$r$ & resource growth rate & 0.91 & \\
\hline$B_{\text {sup }}$ & environmental carrying capacity & 18500 & (tons) \\
\hline$q$ & resource catchability & $4.93 * 10^{-3}$ & $(\text { year })^{-1}$ \\
\hline$d$ & rate of "available" discards & 5.78 & $($ tons $) \cdot(\text { year })^{-1}$ \\
\hline$s$ & "feeding on discards" effect & 2.135 & (bird couples).(tons $)^{-1}$ \\
\hline$F_{0}$ & "natural bird population" & 180 & (bird couples) \\
\hline
\end{tabular}

\section{A.2 Computation of the viability kernel}

We refer to De Lara and Doyen (2008) for the resolution of viability problems in a discrete time framework.

Consider the viability problem defined by the dynamics (1) and the constraints (4) and (5). The aim of the analysis is to define the viability kernel (eq. 6) $\operatorname{Viab}\left(h_{\min }, F_{\min }\right) \subseteq$ $\mathbb{B}=\left[0, B_{\text {sup }}\right]$.

Constraint (4) results in the necessary condition

$$
B_{t} \geq \frac{h_{m i n}}{q}
$$

We define the threshold value $\underline{B}\left(h_{\min }\right)=\frac{h_{\min }}{q}$. The economic constraint (eq. 4$)$ is thus equivalent to the state constraint $B_{t} \geq \underline{B}\left(h_{\min }\right)$. This constraint is stationary through time.

According to Aubin (1991) and De Lara and Doyen (2008), the viability kernel of the problem is the biggest invariant set within the constrained set $\left[\underline{B}\left(h_{\min }\right), B_{\text {sup }}\right]$, in the sense that:

- from any state $B_{0} \in$ Viab there are trajectories staying within Viab and respecting the constraints forever (invariance of the viability kernel)

- from any state $B_{0} \in \mathbb{B} \backslash$ Viab there are no trajectory satisfying the constraint forever.

To prove the results presented in section 3.2, we will proceed as follows: 
- We introduce some preliminary results

- We then show that the whole constrained domain $\left[\underline{B}\left(h_{\min }\right), B_{\text {sup }}\right]$ is viable if condition (7) holds.

- We last prove that the viability kernel is empty if condition (7) does not hold.

\section{Step 1: Preliminary results}

- Given the ecological constraint (5) and the definition (3), any viable decision must satisfy $E_{t} \geq \underline{E}\left(F_{\min }\right)$, with

$$
\underline{E}\left(F_{\min }\right)=\frac{F_{\min }-F_{0}}{s d}
$$

- For any $B_{t} \geq \underline{B}\left(h_{m i n}\right)$, we define the fishing effort $\bar{E}\left(h_{m i n}, B_{t}\right)$ such that $B_{t+1}=$ $\underline{B}\left(h_{\text {min }}\right)$. Given the dynamics $(1)$, it reads

$$
\bar{E}\left(h_{\text {min }}, B_{t}\right)=\frac{1}{q}\left(1+r\left(1-\frac{B_{t}}{B_{\text {sup }}}\right)-\frac{h_{\text {min }}}{q} \frac{1}{B_{t}}\right)
$$

$\bar{E}\left(h_{\min }, B_{t}\right)$ satisfies the three following properties:

i) As the dynamics (1) is decreasing with the effort (the higher $E_{t}$ the lower $\left.B_{t+1}\right)$, for any $B_{t} \in\left[\underline{B}\left(h_{\text {min }}\right), B_{\text {sup }}\right]$, if $E_{t} \leq \bar{E}\left(h_{\text {min }}, B_{t}\right)$ then $B_{t+1} \geq \underline{B}\left(h_{\text {min }}\right)$; respectively, we have: if $E_{t} \geq \bar{E}\left(h_{\min }, B_{t}\right)$ then $B_{t+1} \leq \underline{B}\left(h_{\min }\right)$.

ii) We have $\bar{E}\left(h_{\min }, \underline{B}\left(h_{\min }\right)\right)=\frac{r}{q}\left(1-\frac{h_{\min }}{q B_{\text {sup }}}\right)$ which is equivalent to

$$
r \underline{B}\left(h_{\min }\right)\left(1-\frac{\underline{B}\left(h_{\min }\right)}{B_{\mathrm{sup}}}\right)=q \underline{B}\left(h_{\min }\right) E_{t}
$$

It means that for $B_{t}=\underline{B}$ and $E_{t}=\bar{E}\left(h_{m i n}, \underline{B}\left(h_{m i n}\right)\right)$, the growth of the natural resource is equal to the catches; we have a stationary state, and the resource stock remains at $\underline{B}\left(h_{m i n}\right)$, resulting in an equilibrium.

iii) Note that $\bar{E}\left(h_{\min }, B_{t}\right)$ is an inverted U-shape parabola.

On the interval $\left[\underline{B}\left(h_{\text {min }}\right), B_{\text {sup }}\right]$, its minimum value is at one of the boundary of the definition set.

Standard computation gives $\bar{E}\left(h_{\min }, \underline{B}\left(h_{\min }\right)\right)=\frac{r}{q}\left(1-\frac{h_{\min }}{q B_{s u p}}\right)$, and $\bar{E}\left(h_{\text {min }}, B_{\text {sup }}\right)=\frac{1}{q}\left(1-\frac{h_{\text {min }}}{q B_{\text {sup }}}\right)$.

As $r<1$, we have $\bar{E}\left(h_{\text {min }}, \underline{B}\left(h_{\text {min }}\right)\right)<\bar{E}\left(h_{\text {min }}, B_{\text {sup }}\right)$, which means that

$$
\underset{B \in\left[\underline{B}\left(h_{\text {min }}\right), B_{\text {sup }}\right]}{\arg \min } \bar{E}\left(h_{\text {min }}, B\right)=\underline{B}\left(h_{\text {min }}\right) .
$$


Step 2: Proof that the viability kernel is $\left[\underline{B}\left(h_{\min }\right), B_{\text {sup }}\right]$ when condition $(7)$ holds.

We assume that $F_{\min } \leq \frac{r s d}{q}\left(1-\frac{h_{\min }}{q B_{s u p}}\right)+F_{0}($ condition 7$)$.

From relation (A.1), we know that the Viab $\subseteq\left[\underline{B}\left(h_{\text {min }}\right), B_{\text {sup }}\right]$. To prove our claimed result, we need to prove that $\left[\underline{B}\left(h_{\text {min }}\right), B_{\text {sup }}\right] \subseteq$ Viab, which leads to the equality of the sets. For that purpose, we only have to show to that there exists (at least) one decision rule that keeps the state of the system within the set $\left[\underline{B}\left(h_{\min }\right), B_{\text {sup }}\right]$ while respecting the constraint.

Let us define the following closed-loop decision rule: $E_{t}=\bar{E}\left(h_{m i n}, B_{t}\right)$, defined at step 1 above.

From any initial state $B_{t} \in\left[\underline{B}\left(h_{\text {min }}\right), B_{\text {sup }}\right]$, we have $B_{t+1}=\underline{B}\left(h_{\text {min }}\right)$, by definition of $\bar{E}\left(h_{\min }, B_{t}\right)$. Then, according to the properties ii) of $\bar{E}\left(h_{\min }, \underline{B}\left(h_{\min }\right)\right)$ described at step 1 , the trajectory is stationary at $\underline{B}\left(h_{\min }\right)$.

Along this particular trajectory, as $B_{t} \geq \underline{B}\left(h_{\min }\right)$ for any $t$, the economic constraint (4) is satisfied at any time.

Moreover, along that trajectory, for any time $t$, we have $E_{t}=\bar{E}\left(h_{\min }, B_{t}\right)$.

Using the result iii) exhibited in step 1 that $\bar{E}\left(h_{\min }, B_{t}\right) \geq \bar{E}\left(h_{\min }, \underline{B}\left(h_{\min }\right)\right)$ for all $B \in\left[\underline{B}\left(h_{\text {min }}\right), B_{\text {sup }}\right]$, we have for all times $E_{t} \geq \bar{E}\left(h_{\min }, \underline{B}\left(h_{\min }\right)\right)$, where $\bar{E}\left(h_{\min }, \underline{B}\left(h_{\min }\right)\right)=\frac{r}{q}\left(1-\frac{h_{\min }}{q B_{\text {sup }}}\right)$ (see step 1 , point ii) above).

The condition (7) is equivalent to $\frac{r}{q}\left(1-\frac{h_{\min }}{q B_{s u p}}\right) \geq \frac{F_{\min }-F_{0}}{s d}$.

We thus get $E_{t} \geq \frac{F_{\min }-F_{0}}{s d}$, which implies that the biological constraint (5) is satisfied, according to the result (A.2) presented at step 1.

For any state within $\left[\underline{B}\left(h_{\min }\right), B_{\text {sup }}\right]$, the proposed decision rule leads to a trajectory satisfying the viability constraints at all times.

We thus have $\left[\underline{B}\left(h_{\min }\right), B_{\text {sup }}\right] \subseteq \operatorname{Viab}\left(h_{\min }, F_{\text {min }}\right)$.

Q.E.D.

The viable decisions associated with a given viable states $B \in \operatorname{Viab}\left(h_{\min }, F_{\text {min }}\right)$ are $\underline{E}\left(F_{\min }\right) \leq E(B) \leq \bar{E}\left(h_{\min }, B\right)$. The first inequality is required to the satisfaction of the ecological constraint (see first point of step 1 ), and the second inequality is required to maintain the state $B$ within the viability kernel (see step 1, point i)). 
497

$$
\begin{aligned}
& F_{\text {min }}>\frac{r s d}{q}\left(1-\frac{h_{\text {min }}}{q B_{\text {sup }}}\right)+F_{0} \\
\Leftrightarrow & \frac{F_{\text {min }}-F_{0}}{s d}>\frac{r}{q}\left(1-\frac{h_{\text {min }}}{q B_{\text {sup }}}\right)
\end{aligned}
$$

which means, according to step 1, point ii) above, and to the particular fishing effort $E_{t}=\underline{E}\left(F_{\text {min }}\right)=\frac{F_{\min }-F_{0}}{s d}$ that

$$
\underline{E}\left(F_{\min }\right)>\bar{E}\left(h_{\min }, \underline{B}\left(h_{\min }\right)\right) .
$$

Step 3: Proof that the viability kernel is empty when when condition (7) does

In our model, for $r<1$, if a stationary fishing effort is applied, the biomass reaches an associated equilibrium

$$
B^{s s}(E)=B_{\text {sup }}\left(1-\frac{q}{r} E\right) .
$$

in a finite time (Clark, 1985). Moreover, the higher the fishing effort $E$, the lower the equilibrium biomass.

Let us consider the particular fishing effort $\underline{E}\left(F_{\min }\right)=\frac{F_{\min }-F_{0}}{s d}$, which is the minimal fishing effort such that the ecological constraint (5) is satisfied. From any initial state in the constraint domain $\left[\underline{B}\left(h_{m i n}\right), B_{\text {sup }}\right]$ we assume a stationary fishing effort $E_{t}=\underline{E}\left(F_{\min }\right)$.

If condition (7) doesn't hold, we have

As $\bar{E}\left(h_{\min }, \underline{B}\left(h_{\min }\right)\right)=E^{s s}\left(\underline{B}\left(h_{\min }\right)\right)$ (see step 1, point ii) above), according to eqs. (A.3) and (A.4), we have $B^{s s}\left(\underline{E}\left(F_{\min }\right)\right)<\underline{B}\left(h_{\min }\right)$, whatever the initial condition $B_{0} \in\left[\underline{B}\left(h_{\text {min }}\right), B_{\text {sup }}\right]$. The economic constraint (4) can thus not be satisfied in the long-run.

Note that reducing the fishing effort below $\underline{E}\left(F_{\min }\right)$ would violate the ecological constraint, while increasing it would lead to a lower biomass equilibrium (see point i) of step 1). Non-stationary fishing effort such that $E_{t} \geq \underline{E}\left(F_{\min }\right)$ would lead to lower biomass levels than $B^{s s}\left(\underline{E}\left(F_{\min }\right)\right)$. 


\section{References}

Aubin, J.-P., 1991. Viability theory. Birkhauser, Springer Verlag.

Béné, C., Doyen, L. and Gabay, D., 2001. A viability analysis for a bio-economic model. Ecological Economics, 36:385-396.

Calixto-Albarran, I. and Osorno, J.-L., 2000. The diet of the Magnificent Frigatebird during chick rearing. The Condor, 102:569-576

Commission of the European Communities, 2007. A policy to reduce unwanted bycatches and eliminate discards in European fisheries. Communication from the commission to the Council and the European Parliament. COM(2007) 136 final.

Chapel L., Deffuant G., Martin S. and Mullon C., 2008. Defining yield policies in a viability approach. Ecological Modelling, 212:10-15.

Clark, C.W., 1985. Bioeconomic Modelling and Fisheries Management. John Wiley and Sons: New York.

Cury, P., Mullon, C., Garcia, S. and Shannon, L.J., 2005. Viability theory for an ecosystem approach to fisheries. ICES Journal of Marine Science, 62:577-584.

De Lara, M. and doyen L., 2008. Sustainable Management of Natural Resources: Mathematical Models and Methods. Springer, 266p.

Doyen, L. and Béné, C., 2003. Sustainability of fisheries through marine reserves: a robust modeling analysis. Journal of Environmental Management, 69:1-13.

Doyen L., De Lara M., Ferraris J. and Pelletier D., 2007. Sustainability of exploited marine ecosystems through protected areas: a viability model and a coral reef case study. Ecological Modelling, 208:353-366.

Eisenack, K., Sheffran, J. and Kropp, J., 2006. The Viability Analysis of Management Frameworks for fisheries. Environmental modelling and assessment, 11:6979.

Furness R., 1999. Will reduced discarding help or harm seabird populations? Ecosystem Approaches for Fisheries Management. Alaska Sea Grant College Program. AK-SG-99-0., p. 481-488.

Furness R., 2003. Impacts of fisheries on seabirds communities. Scientia Marina, 67:33-45.

Hilborn, R. and Walters, C., 1992. Quantitative Fisheries Stock Assessment: Choice, Dynamics and Uncertainty. International Thomson Publishing.

Martinet, V., Thébaud, O. and Doyen, L., 2007. Defining viable recovery paths toward sustainable fisheries. Ecological Economics, 64:411-422.

Morris, W.F. and Doak, D.F., 2003. Quantitative Conservation Biology: Theory and Practice of Population Viability Analysis. Sinauer Associates.

Weimerskirch, H., Chastel, O., Barbraud, C. and Tostain, O., 2003. Frigatebirds ride high on thermals. Nature (23 January 2003), 421:333-334. 